\title{
Consumo alimentar de adolescentes com e sem sobrepeso do Município do Rio de Janeiro
}

\author{
Food intake in overweight and normal-weight \\ adolescents in the city of Rio de Janeiro
}

Roseli G. Andrade 1

Rosangela A. Pereira 1

Rosely Sichieri 2

\footnotetext{
1 Instituto de Nutrição Josué de Castro, Centro de Ciências da Saúde, Universidade Federal do Rio de Janeiro. $A v$. Brigadeiro Trompowski $s / n$, Bloco J, Rio de Janeiro, RJ 21941-590, Brasil.

2 Instituto de Medicina Social, Universidade do Estado do Rio de Janeiro. Rua São Francisco Xavier, 524, Bloco E, Rio de Janeiro, RJ 20550-012, Brasil. sichieri@uerj.br
}

\begin{abstract}
This article compares the food intake of overweight and normal-weight adolescents from 12 to 17.9 years of age (71.8\% of the original sample). Data were obtained in a survey performed in the city of Rio de Janeiro, Brazil, in 1995-1996. Food intake was assessed by a semiquantitative food frequency questionnaire, and nutritional status was assessed by body mass index $\left(B M I=\right.$ weight/stature $\left.{ }^{2}\right)$. Adolescents classified as overweight reached the 90th percentile of the 1989 Brazilian BMI distribution. Food intake of overweight and non-overweight subjects was similar. Both groups showed below-minimum-recommended food-group intakes and higher intake of high-density food items. These nutritional habits are risk factors for obesity and chronic diseases later in life.
\end{abstract}

Key words Adolescents; Body Mass Index; Nutritional Status; Food Habits

Resumo Comparam-se, neste estudo, as características do consumo alimentar de uma amostra probabilística de adolescentes com sobrepeso e adolescentes com peso normal, com idades entre 12 e 17,9 anos. Os dados analisados foram obtidos em pesquisa realizada no Município do Rio de Janeiro, em 1995-1996. Estimou-se o consumo alimentar através de um questionário de freqüencia do consumo de alimentos semiquantitativo. Avaliou-se o estado nutricional através do indice de massa corporal (IMC = peso/altura ${ }^{2}$ ), classificando-se os adolescentes com e sem sobrepeso pelo percentil 90 da distribuição do IMC de crianças brasileiras de 1989. O consumo de indivíduos com e sem sobrepeso foi semelhante, em ambos os grupos observou-se consumo inferior ao mínimo das porções recomendadas dos grupos alimentares e consumo elevado de alimentos de alta densidade energética. Esses hábitos alimentares propiciam o desenvolvimento de excesso de peso e podem ser determinantes de obesidade e doenças crônicas não transmissíveis na maturidade.

Palavras-chave Adolescente; Índice de Massa Corporal; Estado Nutricional; Hábitos Alimentares 


\section{Introdução}

O sobrepeso e a obesidade em adolescentes são fatores de risco para doenças crônicas na idade adulta (Jacobson, 2001). Tipicamente, o sobrepeso tem sido definido como excesso de massa corpórea ou de peso relativo, quando se considera uma distribuição referencial abrangendo idade e sexo (Himes \& Dietz, 1994). Idealmente, as definições de excesso de peso e obesidade deveriam basear-se em critérios de saúde, notadamente nas estatísticas de morbidade e mortalidade. Entretanto, existem poucos estudos abordando estes aspectos em relação ao excesso de peso na adolescência (Jacobson, 2001).

Tem-se utilizado o índice de massa corporal (IMC, obtido pela divisão da massa corporal pela estatura elevada ao quadrado) como indicador de sobrepeso ou obesidade em adolescentes. Seu uso justifica-se pela importante correlação com a gordura corporal total e subcutânea dos adolescentes, com a pressão arterial e com o perfil de lipídios séricos tanto na adolescência quanto na vida adulta (Himes \& Dietz, 1994). O IMC é um método de avaliação nutricional de baixo custo e fácil realização, apesar de ter como limitação a não distinção dos diferentes componentes da massa corporal (água, massa muscular, massa adiposa). Outra limitação no uso do IMC para avaliação nutricional de adolescentes é que, diferentemente do adulto, no jovem não se utilizam pontos de corte estabelecidos com base na predição de morbidades ou mortalidade. Na avaliação do estado nutricional de adolescentes, comparase o IMC com uma distribuição de referência, dos percentis dos IMC por sexo e faixa etária (Engstrom et al., 1998).

No Brasil, nas últimas décadas, a prevalência da obesidade tem-se elevado nos diferentes grupos etários e sociais, como revelam os dados de pesquisas recentes (Coitinho et al., 1991; Pereira, 1998). Essas mudanças vêm ocorrendo paralelamente a modificações expressivas no padrão alimentar da população urbana brasileira. Particularmente, no que se refere à redução no consumo de cereais, leguminosas, raízes e tubérculos, à substituição da gordura animal pelos óleos vegetais, bem como ao aumento no consumo de ovos e de leite e derivados. Tais alterações tiveram como conseqüência a redução da participação relativa dos carboidratos na dieta e um aumento da participação dos lipídios, havendo, também, um aumento da proporção de proteínas de origem animal e dos lipídios de origem vegetal (Mondini \& Monteiro, 1994; Monteiro et al., 2000b). Essas mudanças no consumo alimentar, em conjunto com outras alterações no estilo de vida - especialmente aquelas relacionadas ao nível de atividade física - podem ter contribuído para a elevação das taxas de prevalência do excesso de peso (Sichieri, 1998).

Comparam-se, aqui, as características do consumo alimentar de adolescentes com e sem sobrepeso, em uma amostra probabilística de moradores do Município do Rio de Janeiro.

\section{Material e métodos}

Analisam-se, neste estudo as informações sobre consumo de alimentos de 387 adolescentes com idades entre 12 e 17,9 anos com e sem sobrepeso, que correspondem a $86,5 \%$ da amostra inicial. De um total de 554 indivíduos entrevistados nessa faixa etária, foram excluídos das análises $49(8,8 \%)$, para os quais não estavam disponíveis as informações sobre dados antropométricos. Foram também excluídos 58 adolescentes $(10,5 \%)$ com baixo peso, perfazendo um total de 447 indivíduos elegíveis para este estudo. Desse grupo, foram excluídos 11 (2,5\%) indivíduos para os quais não se dispunha de dados sobre consumo de alimentos e outros 49 $(11,0 \%)$ registros que relatavam consumo energético superior a 6 mil kcal.

Conforme detalhada descrição de Sichieri \& Silva (1998), o processo de amostragem adotado na Pesquisa Nutrição e Saúde no Rio de Janeiro foi o de conglomerados em dois estágios, com probabilidades de seleção proporcionais aos tamanhos dos setores censitários, definidos pela Fundação Instituto Brasileiro de Geografia e Estatística (IBGE) para o Município do Rio de Janeiro, Brasil. Sessenta setores censitários foram sorteados no primeiro estágio. Em seguida, procedeu-se à atualização do número de domicílios nos setores sorteados e, então, à seleção sistemática de 34 domicílios em cada um desses setores. Dos 2.040 domicílios elegíveis, 1.668 participaram da pesquisa (taxa de não-resposta $=18,2 \%$ ).

Os dados foram coletados entre novembro de 1995 e setembro de 1996 por entrevistadores e antropometristas treinados. A equipe elaborou manuais e orientações escritas para os procedimentos adotados na coleta de dados e treinou os profissionais envolvidos na pesquisa.

Pôde-se estimar o consumo alimentar por meio de um questionário de freqüência do consumo de alimentos semiquantitativo com uma lista de 80 itens alimentares, previamente validado em adultos moradores no Rio de Janeiro (Sichieri \& Everhart, 1998). Embora o Questionário de Freqüência de Consumo Alimentar 
(QFCA) utilizado tenha sido elaborado e validado para adultos, foi aplicado previamente em outra pesquisa com adolescentes (Fonseca et al., 1998) quando foi desenvolvido um préteste que levou à inclusão de pipoca, pizza e hambúrguer, itens de consumo freqüente entre adolescentes e que não estavam incluídos no questionário original.

Para a avaliação do estado nutricional, consideraram-se as medidas de peso e altura. Para a tomada do peso, utilizou-se balança digital marca Filizola, quando o examinando permanecia com o mínimo de roupa possível e descalço. Aferiu-se a altura com o uso de fita métrica aderida a uma parede sem rodapés e um esquadro de madeira, observando-se as recomendações de Lohman et al. (1988).

\section{Análise de dados}

Os adolescentes foram classificados segundo o percentil 90 da distribuição do IMC de crianças brasileiras obtida na Pesquisa Nacional sobre Saúde e Nutrição (Sichieri \& Allam, 1996) em dois grupos: com e sem sobrepeso.

Estimaram-se as médias, com os respectivos erros-padrão, e intervalos de confiança de $95 \%$, da ingestão de gorduras totais, gordura saturada, vitamina A, vitamina C, cálcio e ferro. Selecionaram-se estes nutrientes por sua relevância na saúde coletiva. A anemia e a hipovitaminose A são carências nutricionais cuja expressiva prevalência tem sido enfatizada em algumas pesquisas (Fujimori et al., 1996; Gonçalves-Carvalho et al., 1995), a vitamina C, pelo seu efeito antioxidante e imunológico, o cálcio e sua relação direta com o desenvolvimento osteoarticular e osteoporose, a energia e gorduras, dada a relação com as doenças cardiovasculares e a obesidade.

Para a análise dos dados de consumo de alimentos, desenvolveu-se um programa específico que, inicialmente, transforma as freqüências do consumo de alimentos em freqüências diárias; em seguida, estima, para cada alimento, a quantidade consumida por dia; finalmente, calcula a ingestão de energia utilizando o banco de dados de composição de alimentos do Programa de Apoio à Nutrição, desenvolvido pela Escola Paulista de Medicina, Universidade Federal de São Paulo (EPM, 1995).

A composição dos alimentos não incluídos nesse banco de dados - por exemplo, inhame foram obtidas na Tabela de Composição dos Alimentos, utilizada pelo Estudo Nacional da Despesa Familiar (ENDEF), 1974-1975 (IBGE, 1985). Para dados sobre conteúdo de ácidos graxos dos alimentos, utilizou-se, complementarmente, a publicação Food Values of Portions Commonly Used (Pennington, 1989). Para alimentos como bolacha e macarrão, cujos valores norte americanos baseiam-se em produtos enriquecidos - o que não é o mais usual no Brasil - utilizamos os dados do ENDEF para ferro e vitaminas A e C (Sichieri, 1998).

Pelo fato de se constituírem eventos muito raros, ou quase impossíveis de ocorrer, foram excluídos os casos com consumo energético superior a 6 mil kcal ou inferior a $500 \mathrm{kcal}$.

Analisou-se também o consumo de produtos alimentares considerados de alta densidade energética. A densidade energética refere-se à quantidade de energia fornecida por grama de peso do alimento. Os produtos que fornecem mais de $1,5 \mathrm{kcal}$ por grama de peso são considerados de densidade energética elevada (Rolls et al., 1999). Os itens alimentares de alta densidade energética incluídos no QFCA aplicado estão discriminados nas Tabelas 1 e 2 .

Para estimar a adequação do consumo de grupos de alimentos utilizou-se a recomendação de Sichieri et al. (2000) para o consumo dos grupos das frutas, leite e derivados, feijões e grãos, arroz e feculentos e das hortaliças. Para estimar a adequação do consumo das carnes utilizaram-se os valores determinados pelo Food Guide Pyramid (Welsh et al., 1992), porque a publicação nacional não contemplava o grupo das carnes.

Para classificar o consumo alimentar dos adolescentes em abaixo ou acima das recomendações, foi preciso comparar com as recomendações as porções consumidas de todos os alimentos incluídos em cada um dos Grupos de Alimentos considerados. Verificou-se que as porções estipuladas no questionário para os itens alimentares, em média, não se distanciavam do preconizado por Sichieri et al. (2000) nas recomendações. O questionário avalia item por item de consumo enquanto que nas recomendações os autores definiram um alimento/porção como o “equivalente básico” para representar cada grupo. As porções estipuladas no questionário e as recomendadas para os grupos de alimentos foram semelhantes. Por exemplo, a recomendação para feijões e grãos é de 4 porções de $70 \mathrm{~g}$ de feijão, ao passo que, no questionário, a porção padronizada equivale a $80 \mathrm{~g}$ de feijão. As demais porções também eram mais ou menos equivalentes, com exceção das frutas que, no questionário, aparecem em porções maiores. O estudo de validação (Sichieri \& Everhart, 1998) indicou que o QFCA utilizado superestima o consumo de frutas, em decorrência da inclusão de um razoável núme- 
Tabela 1

Contribuição percentual média (e intervalos de confiança de 95\%) para o consumo total de energia dos itens alimentares de alta densidade energética e de bebidas, meninos adolescentes com e sem sobrepeso.

Rio de Janeiro, Brasil, 1995-1996.

\begin{tabular}{|c|c|c|c|c|}
\hline \multirow{2}{*}{$\begin{array}{l}\text { Item alimentar } \\
\text { (densidade energética) }\end{array}$} & \multicolumn{2}{|c|}{ Meninos sem sobrepeso $(n=151)$} & \multicolumn{2}{|c|}{ Meninos com sobrepeso $(n=63)$} \\
\hline & $\%$ & $\begin{array}{l}\text { (Intervalo } \\
\text { de confiança) }\end{array}$ & $\%$ & $\begin{array}{l}\text { (Intervalo } \\
\text { de confiança) }\end{array}$ \\
\hline Achocolatados $(3,92)$ & 0,2 & $(0,2-0,3)$ & 0,3 & $(0,1-0,5)$ \\
\hline Açúcar $(3,90)$ & 5,2 & $(4,4-6,0)$ & 5,7 & $(4,7-6,6)$ \\
\hline Atum/sardinha $(1,97)$ & 0,1 & $(0,0-0,1)$ & 0,0 & $(0,0-0,1)$ \\
\hline Bacon, toucinho $(5,73)$ & 0,1 & $(0,0-0,2)$ & 0,7 & $(0,2-1,6)^{1}$ \\
\hline Balas $(3,98)$ & 0,1 & $(0,0-0,1)$ & 0,1 & $(0,0-0,1)$ \\
\hline Batata frita ou chips $(5,23)$ & 2,6 & $(1,8-3,3)$ & 3,3 & $(2,0-4,6)$ \\
\hline Biscoito doce $(4,00)$ & 1,0 & $(0,6-1,3)$ & 1,2 & $(0,5-1,9)$ \\
\hline Bolo $(3,50)$ & 1,8 & $(1,2-2,4)$ & 1,8 & $(1,0-2,6)$ \\
\hline Cerveja & 0,9 & $(0,5-1,3)$ & 1,3 & $(0,7-2,0)$ \\
\hline Chocolate (em barra, bombom) $(5,20)$ & 0,8 & $(0,4-1,1)$ & 0,6 & $(0,3-0,9) 1$ \\
\hline Hambúrguer $(2,76)$ & 1,3 & $(0,9-1,6)$ & 1,7 & $(1,1-2,3)$ \\
\hline Lingüiça, salsicha $(3,69)$ & 0,5 & $(0,3-0,7)$ & 0,3 & $(0,2-0,5) 1$ \\
\hline Maionese $(3,80)$ & 0,2 & $(0,1-0,2)$ & 0,4 & $(0,2-0,5)^{1}$ \\
\hline Manteiga $(7,17)$ & 1,4 & $(1,2-1,5)$ & 1,5 & $(1,2-1,8)$ \\
\hline Mate $(2,06)$ & 0,2 & $(0,1-0,3)$ & 0,1 & $(0,1-0,2)$ \\
\hline Outras bebidas alcoólicas $(2,31)$ & 0,1 & $(0,0-0,1)$ & 0,3 & $(-0,2-0,8)$ \\
\hline Pão doce $(2,40)$ & 0,5 & $(0,2-0,7)$ & 0,5 & $(0,3-0,8)$ \\
\hline Pipoca $(4,60)$ & 0,4 & $(0,2-0,7)$ & 0,3 & $(0,1-0,4)$ \\
\hline Pizza $(2,36)$ & 1,1 & $(0,7-1,5)$ & 1,3 & $(0,6-2,0)$ \\
\hline Pudins, doces e compotas $(2,32)$ & 0,8 & $(0,3-1,2)$ & 0,7 & $(0,3-1,1)$ \\
\hline Refrigerante & 2,1 & $(1,7-2,5)$ & 2,2 & $(1,6-2,8)$ \\
\hline Salgados: quibe, pastel $(4,43)$ & 1,3 & $(0,9-1,7)$ & 1,3 & $(0,8-1,9)$ \\
\hline Sorvetes $(1,91)$ & 1,1 & $(0,5-1,7)$ & 1,1 & $(0,5-1,6)$ \\
\hline Vinho & 0,4 & $(0,1-0,7)$ & 0,0 & $(0,0-0,0)$ \\
\hline
\end{tabular}

1 valor de $p<0,05$

ro de frutas e do tamanho estimado das porções padronizadas. Portanto, não há por que pressupor erro diferencial entre os sexos ou entre classificação do estado nutricional.

Para definir o consumo diário dos grupos de alimentos, somaram-se as porções diárias consumidas de cada um dos alimentos incluídos nos diversos grupos (por exemplo, porção diária consumida de feijão + porção diária consumida de lentilha/ ervilha = total da porção diária consumida de feijões e grãos). Em seguida, para classificar o consumo em acima/abaixo da recomendação (discriminada na Tabela 3), comparou-se a quantidade de porções consumidas dos grupos com o número de porções do "equivalente básico" recomendado para cada grupo, criando uma variável dicotômica, onde o valor " 0 " correspondia ao consumo igual ou acima da recomendação (isto é, consumo adequado) e o valor " 1 " referia-se ao consumo abaixo da recomendação (ou seja, inadequado), obtendo-se, dessa forma, a proporção de indivíduos com consumo abaixo do recomendado (inadequado) que foi representada graficamente (Figura 1).

A análise expandiu as estimativas populacionais, considerando a probabilidade de seleção dos domicílios. Os fatores de expansão foram também ajustados segundo as taxas de não-resposta. As estimativas foram obtidas utilizando-se o módulo CSAMPLE do programa Epi Info. Para testar a diferença entre as médias dos parâmetros avaliados utilizou-se o teste $\mathrm{t}$ de Student (bicaudal).

\section{Resultados}

A prevalência de sobrepeso entre os meninos foi $29,3 \%$ e, entre as meninas, em torno de 


\begin{tabular}{|c|c|c|c|c|}
\hline \multirow{2}{*}{$\begin{array}{l}\text { Item alimentar } \\
\text { (densidade energética) }\end{array}$} & \multicolumn{2}{|c|}{ Meninas sem sobrepeso $(n=146)$} & \multicolumn{2}{|c|}{ Meninas com sobrepeso $(n=27$} \\
\hline & & $\begin{array}{l}\text { (Intervalo } \\
\text { de confiança) }\end{array}$ & $\%$ & $\begin{array}{l}\text { (Intervalo } \\
\text { de confiança) }\end{array}$ \\
\hline Achocolatados $(3,92)$ & 0,2 & $(0,1-0,3)$ & 0,1 & $(0,0-0,2)$ \\
\hline Açúcar $(3,90)$ & 4,8 & $(4,1-5,5)$ & 4,2 & $(2,8-5,6)$ \\
\hline Atum/sardinha $(1,97)$ & 0,1 & $(0,0-0,1)$ & 0,0 & $(0,0-0,1)$ \\
\hline Bacon, toucinho $(5,73)$ & 0,7 & $(0,5-1,5)$ & 0,2 & $(0,0-0,5)^{1}$ \\
\hline Biscoito doce $(4,00)$ & 1,1 & $(0,7-1,6)$ & 1,1 & $(0,5-1,8)$ \\
\hline Balas $(3,98)$ & 0,1 & $(0,1-0,2)$ & 0,1 & $(0,0-0,2)$ \\
\hline Batata frita ou chips $(5,23)$ & 4,0 & $(2,7-5,3)$ & 5,8 & $(3,1-8,6)$ \\
\hline Bolo $(3,50)$ & 1,5 & $(1,0-2,0)$ & 1,3 & $(0,6-2,1)$ \\
\hline Cerveja & 1,2 & $(0,6-1,8)$ & 0,6 & $(-0,1-1,2)$ \\
\hline Chocolate (em barra, bombom) $(5,20)$ & 0,4 & $(0,2-0,6)$ & 0,8 & $(-0,2-1,8)$ \\
\hline Hambúrguer $(2,76)$ & 1,8 & $(1,2-2,4)$ & 0,9 & $(-0,1-2,0)$ \\
\hline Lingüiça, salsicha $(3,69)$ & 0,9 & $(0,5-1,3)$ & 1,1 & $(0,6-1,6)$ \\
\hline Maionese $(3,80)$ & 0,3 & $(0,2-0,4)$ & 0,2 & $(0,1-0,4)$ \\
\hline Manteiga $(7,17)$ & 1,5 & $(1,2-1,7)$ & 1,5 & $(1,1-1,9)$ \\
\hline Mate $(2,06)$ & 0,1 & $(0,0-0,1)$ & 0,0 & $(0,0-0,1)$ \\
\hline Outras bebidas alcoólicas $(2,31)$ & 0,1 & $(-0,1-0,3)$ & 0,0 & - \\
\hline Pão doce $(2,40)$ & 0,9 & $(0,2-1,5)$ & 0,5 & $(0,1-0,8)$ \\
\hline Pipoca $(4,60)$ & 0,5 & $(0,3-0,7)$ & 0,2 & $(0,0-0,4)^{1}$ \\
\hline Pizza $(2,36)$ & 1,3 & $(1,0-1,7)$ & 1,5 & $(0,9-2,3)^{1}$ \\
\hline Pudins, doces e compotas $(2,32)$ & 0,9 & $(0,0-1,7)$ & 0,2 & $(0,1-0,3)$ \\
\hline Refrigerante & 2,6 & $(2,0-3,2)$ & 1,9 & $(1,0-2,8)^{1}$ \\
\hline Salgados: quibe, pastel $(4,43)$ & 1,6 & $(1,2-2,1)$ & 1,8 & $(0,7-3,0)$ \\
\hline Sorvetes $(1,91)$ & 0,9 & $(0,6-1,3)$ & 0,6 & $(0,0-1,3)$ \\
\hline Vinho & 0,0 & $(0,0-0,0)$ & 0,1 & $(-0,1-0,3)$ \\
\hline
\end{tabular}

* valor de $p<0,05$

15,0\%. A análise do consumo de nutrientes em adolescentes com e sem sobrepeso é apresentada na Tabela 4. Os meninos com sobrepeso apresentaram consumo médio de energia ligeiramente mais elevado do que os meninos que não tinham sobrepeso. No entanto, o consumo total de gorduras foi maior entre os meninos sem sobrepeso.

Entre as meninas com e sem sobrepeso, não se observaram diferenças significativas no consumo de energia. O consumo de gordura foi mais elevado no grupo com sobrepeso. Para os demais nutrientes avaliados, não houve diferença entre os grupos (Tabela 4).

Os meninos com sobrepeso apresentaram maior consumo médio de porções do grupo do arroz e feculentos do que os meninos sem sobrepeso (valor de $\mathrm{p}<0,05$ ), e consumo similar de hortaliças, feijões e grãos e leite e derivados. Meninas com sobrepeso apresentaram o con- sumo médio menor de porções de arroz e feculentos, feijões e grãos, hortaliças e leite e derivados (valor de $\mathrm{p}<0,05$ ) (Figura 2).

Meninos sem sobrepeso apresentaram maior prevalência de ingestão do grupos do leite e derivados e arroz e feculentos em porções abaixo do recomendado. Meninas sem sobrepeso apresentaram prevalência mais elevada de consumo abaixo do mínimo de porções recomendadas para o grupo das frutas (Figura 1).

A contribuição para o consumo total de energia dos produtos de alta densidade energética e bebidas foi de $25,9 \%$, para os meninos com sobrepeso, e $26,1 \%$, para aqueles com peso normal, de $26,1 \%$ entre as meninas com sobrepeso e $26,6 \%$ para as sem sobrepeso (diferenças não significativas estatisticamente).

Meninos com sobrepeso referiram consumir bacon e maionese em maior proporção do que os meninos com peso dentro da faixa de 
Tabela 3

\begin{tabular}{|c|c|c|c|}
\hline Grupo & Itens incluídos & $\begin{array}{l}\text { Quantidade da } \\
\text { porção equivalente }\end{array}$ & $\begin{array}{l}\text { Recomendações } \\
\text { para adolescentes } \\
\text { (porções) }\end{array}$ \\
\hline Leite e derivados & $\begin{array}{l}\text { Leite, queijo, requeijão } \\
\text { e iogurte }\end{array}$ & $200 \mathrm{ml}$ de leite & 3 \\
\hline Arroz e feculentos & $\begin{array}{l}\text { Arroz, biscoito doce, biscoito } \\
\text { salgado, bolo, farinha, macarrão, } \\
\text { pão doce, pão francês, angu, } \\
\text { aipim, batata, inhame, milho }\end{array}$ & $50 \mathrm{~g}$ de arroz & 8 \\
\hline Feijões e grãos & Feijão, ervilha e lentilha & $70 \mathrm{~g}$ de feijão & 4 \\
\hline Hortaliças & $\begin{array}{l}\text { Chuchu, couve, repolho, chicória, } \\
\text { tomate, abóbora, abobrinha, vagem, } \\
\text { quiabo, cenoura, beterraba, couve-flor }\end{array}$ & $50 \mathrm{~g}$ de chuchu & 5 \\
\hline Frutas & $\begin{array}{l}\text { Abacate, abacaxi, banana, goiaba, } \\
\text { laranja, maçã, mamão, manga, melão, } \\
\text { pêra, uva, maracujá, limão }\end{array}$ & $100 \mathrm{~g}$ laranja & 5 \\
\hline Carnes & $\begin{array}{l}\text { Bife, frango, camarão, carré (carne } \\
\text { de porco), lingüiça, peixe enlatado, } \\
\text { peixe, rabada, vísceras, ovo }\end{array}$ & $50 \mathrm{~g}$ de bife & 3 \\
\hline
\end{tabular}

normalidade e referiram chocolate em menor proporção. Os alimentos de alta densidade energética que mais contribuíram para o consumo total de energia de meninos com e sem sobrepeso foram: açúcar, batata-frita e refrigerantes (Tabela 1).

Com relação ao consumo de alimentos de alta densidade, as meninas com sobrepeso apresentaram consumo proporcionalmente mais elevado de pizza. Contudo, as meninas sem sobrepeso consumiam em maior quantidade $b a$ con, pipoca e refrigerantes. Os alimentos de alta densidade energética que mais contribuíram para o consumo total de energia de meninas também foram batata-frita, açúcar e refrigerantes (Tabela 2 ).

\section{Discussão}

As análises de consumo alimentar, em geral, baseiam-se na avaliação da composição química da dieta. Neste trabalho, avaliamos tanto o consumo de nutrientes quanto a caracterização dos alimentos consumidos, no sentido de identificar padrões de consumo que poderiam explicar o sobrepeso. Padrões de consumo têm sido largamente utilizados na avaliação de die- tas, especialmente no estudo de sua associação com doenças (Helsing, 1995).

No grupo investigado, a prevalência de sobrepeso foi maior entre os meninos. Fonseca et al. (1998), estudando um grupo de adolescentes de classe média na cidade de Niterói, no Estado do Rio de Janeiro, também encontraram prevalência de sobrepeso maior entre os meninos, quando avaliaram o estado nutricional segundo o IMC e a composição corporal obtida através de dobras cutâneas. Entretanto, Veiga et al. (2001) estimou a prevalência da obesidade em estudantes entre 10 e 18 anos de idade Niterói com base no percentual de gordura obtido através da bioimpedância e observou que em meninos a prevalência de obesidade foi $12,8 \%$, versus $19,9 \%$ nas meninas. A autora discute que os diferenciais encontrados nas prevalências de obesidade obtidas através dos diversos métodos podem ser explicados pela interferência da massa muscular, maior nos meninos e que não é estimada com acurácia através do IMC.

Com esta análise, demonstrou-se que o consumo de alimentos entre os adolescentes do Rio de Janeiro caracterizou-se por uma ingestão elevada (aproximadamente a quarta parte do consumo energético total) de produtos de 
Consumo de porções dos grupos de alimentos abaixo do mínimo recomendado, adolescentes sem e com sobrepeso. Município do Rio de Janeiro, Brasil, 1995-1996.1
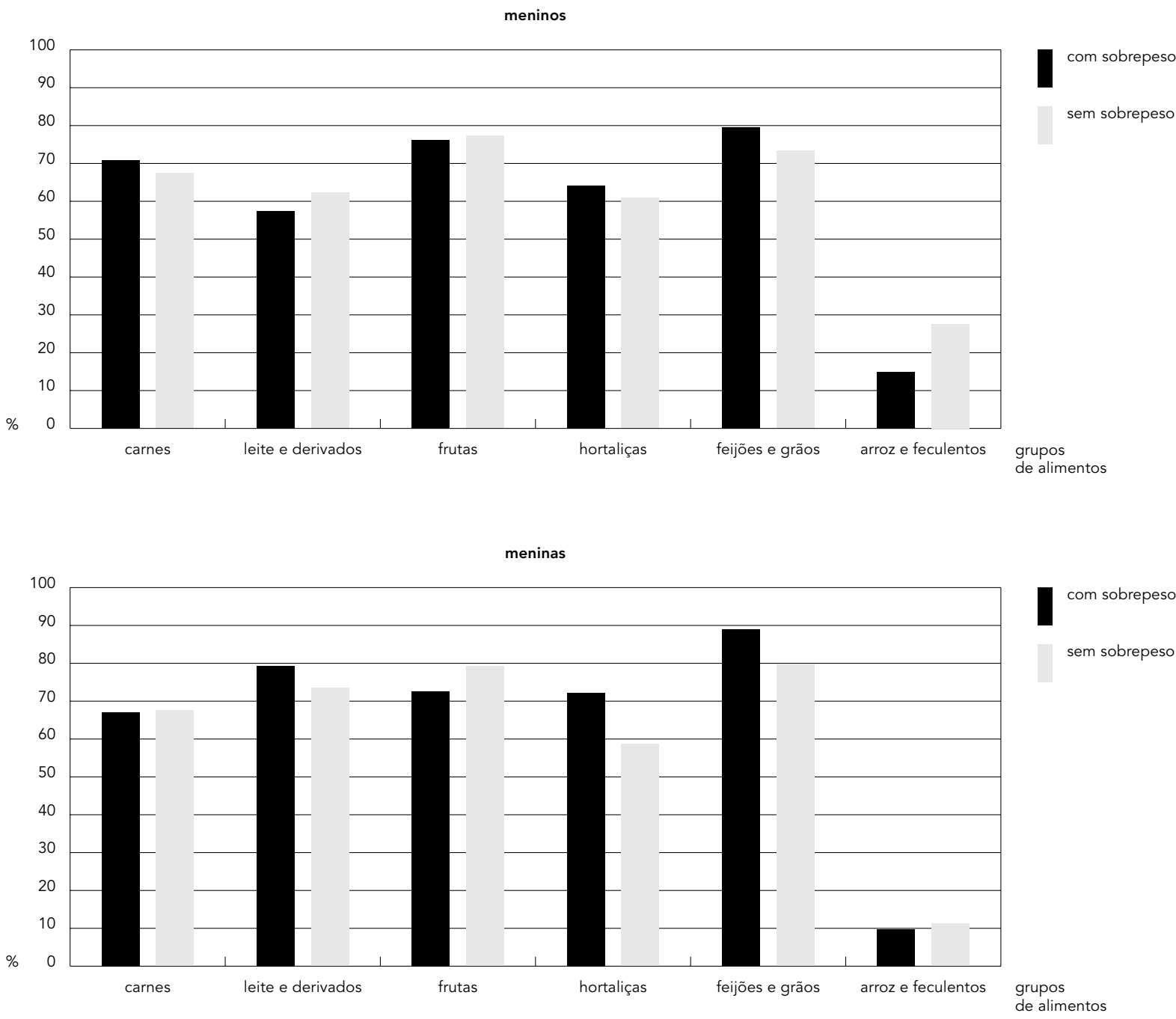

$1100 \%$ equivale ao total de meninos e de meninas em cada categoria.

alta densidade energética. Contudo, o consumo de indivíduos com sobrepeso não apresentou grandes diferenças qualitativas em relação àqueles que tinham peso normal.

O consumo energético dos meninos com sobrepeso foi maior do que o consumo dos sem sobrepeso, mas essa associação não foi observada entre as meninas. Entre as meninas, aquelas com sobrepeso tiveram ingestão média de gordura ligeiramente maior do que as meninas com peso normal. Este dado pode ser o reflexo de uma redução no consumo entre as meninas tendo em vista a diminuição do peso ou, ainda, poderia estar refletindo um relato influenciado pelo conhecimento prévio dos princípios de uma alimentação adequada. Para a análise por grupos de alimentos, nos dois sexos e em ambos os grupos, observou-se freqüência de consumo inferior ao mínimo das porções recomendadas, particularmente de carnes e feijões. O consumo de alimentos de alta densidade energética foi também elevado em ambos os sexos, sem diferenças importantes no grupo com sobrepeso. 
Tabela 4

Médias e intervalos de confiança de $95 \%$ de consumo de nutrientes e energia em adolescentes com e sem sobrepeso.

Município do Rio de Janeiro, Brasil, 1995-1996.

\begin{tabular}{|c|c|c|c|c|}
\hline \multirow[t]{2}{*}{ Nutriente/energia } & \multicolumn{2}{|c|}{ Meninos } & \multicolumn{2}{|c|}{ Meninas } \\
\hline & $\begin{array}{c}\text { Sem sobrepeso }(n=151) \\
\text { Média (Intervalo } \\
\text { de confiança) }\end{array}$ & $\begin{array}{c}\text { Com sobrepeso }(n=63) \\
\text { Média (Intervalo } \\
\text { de confiança) }\end{array}$ & $\begin{array}{c}\text { Sem sobrepeso }(n=146) \\
\text { Média (Intervalo } \\
\text { de confiança) }\end{array}$ & $\begin{array}{c}\text { Com sobrepeso }(n=27) \\
\text { Média (Intervalo } \\
\text { de confiança) }\end{array}$ \\
\hline \multirow[t]{2}{*}{ Energia (kcal) } & 2.697 & 2.8901 & 2.920 & 2.848 \\
\hline & $(2.470-2.923)$ & $(2.655-3.124)$ & $(2.727-3.113)$ & $(2.378-3.318)$ \\
\hline \multirow[t]{2}{*}{ Gordura total (g) } & 84,1 & 76,91 & 87,9 & 93,01 \\
\hline & $(77,4-90,9)$ & $(64,8-89,1)$ & $(82,0-93,8)$ & $(70,4-115,6)$ \\
\hline \multirow[t]{2}{*}{ Gordura saturada (g) } & 29,7 & 27,2 & 31,4 & 33,0 \\
\hline & $(27,3-32,0)$ & $(22,5-31,9)$ & $(28,7-34,0)$ & $(23,8-42,2)$ \\
\hline \multirow[t]{2}{*}{ Cálcio (mg) } & 1.087 & 929 & 1.151 & 1.255 \\
\hline & $(998-1.176)$ & $(783-1.074)$ & $(1.023-1.280)$ & $(977-1.534)$ \\
\hline \multirow[t]{2}{*}{ Ferro (mg) } & 18,8 & 17,1 & 19,1 & 20,8 \\
\hline & $(16,8-20,7)$ & $(15,4-18,8)$ & $(17,5-20,6)$ & $(16,6-25,0)$ \\
\hline \multirow[t]{2}{*}{ Vitamina A (UI) } & 11.982 & 7.7721 & 10.802 & 14.334 \\
\hline & $(9.779-14.185)$ & $(6.131-9.412)$ & $(8.539-13.065)$ & (9.077-19.592) \\
\hline \multirow[t]{2}{*}{ Vitamina C (mg) } & 401,7 & 311,21 & 403,8 & 499,51 \\
\hline & $(347,0-456,4)$ & $(246,2-376,2)$ & $(325,0-482,6)$ & $(353,3-645,7)$ \\
\hline
\end{tabular}

1 valor de $p$ associados ao teste $t<0,05$

$\mathrm{UI}=$ Unidades Internacionais.

Em alguns estudos tem sido demonstrado consumo de indivíduos obesos semelhante ou menor ao de indivíduos eutróficos, revelando uma tendência dos obesos de subestimar o próprio consumo (Fonseca et al., 1998; Ortega et al., 1995; Veiga, 1992; Whitehead \& Paul, 1995). Story et al. (1986) analisaram as medidas antropométricas e a ingestão dietética de adolescentes e não encontraram diferenças significativas entre a ingestão energética entre os indivíduos com peso normal e aqueles com sobrepeso. A relação entre consumo alimentar e obesidade dificilmente é evidenciada em estudos transversais.

Fonseca et al. (1998) observaram menores ingestões de todos os nutrientes nos adolescentes com sobrepeso e concluíram que é bastante difundida, entre as meninas, a "mentalidade dietética” evidenciada, por exemplo, na prática de evitar alimentos energéticos ou na omissão de uma ou mais refeições diárias. Assinale-se que tal comportamento necessariamente não é acompanhado da redução do peso, tendo em vista que o organismo tende a se adaptar a uma redução da ingestão de alimentos diminuindo o gasto de energia e, também, porque, na prática, o que pode estar ocorrendo é a substituição de calorias provenientes de alimentos tradicionais (como o arroz e o feijão) por produtos de baixo valor nutricional e alto conteúdo calórico como os industrializados (refrigerantes, biscoitos, chocolate, sorvete) e pratos que gozam de popularidade entre as gerações mais jovens (batata frita, hambúrguer, cachorro-quente, pizza, salgadinhos), considerando-se o consumo inferior ao recomendado dos principais grupos alimentares e a contribuição dos alimentos de alta densidade energética para o consumo total de energia.

Ortega et al. (1995), através de recordatório de cinco dias, não encontraram diferenças na ingestão energética total entre adolescentes com peso normal e com sobrepeso, porém observaram um consumo de gorduras maior em indivíduos com sobrepeso.

Summerbell et al. (1996) analisaram a relação entre IMC e padrão alimentar, por um recordatório dietético de sete dias, em quatro grupos etários, sendo um dos grupos de adolescentes de 13-14 anos. Neste grupo, observaram uma subestimação do consumo de lanches e da ingestão calórica pelos obesos, principalmente entre as meninas. Esses autores discutem que, provavelmente, o IMC elevado esteja relacionado a uma dieta rica em gorduras e pobre em açúcares.

Segundo resultados encontrados neste trabalho, um dos itens de alta densidade energética que mais contribui para o consumo de energia das meninas foi a batata frita. Particular- 
Consumo diário médio de porções dos grupos de alimentos comparado com as recomendações, adolescentes sem e com sobrepeso. Município do Rio de Janeiro, Brasil, 1995-1996.

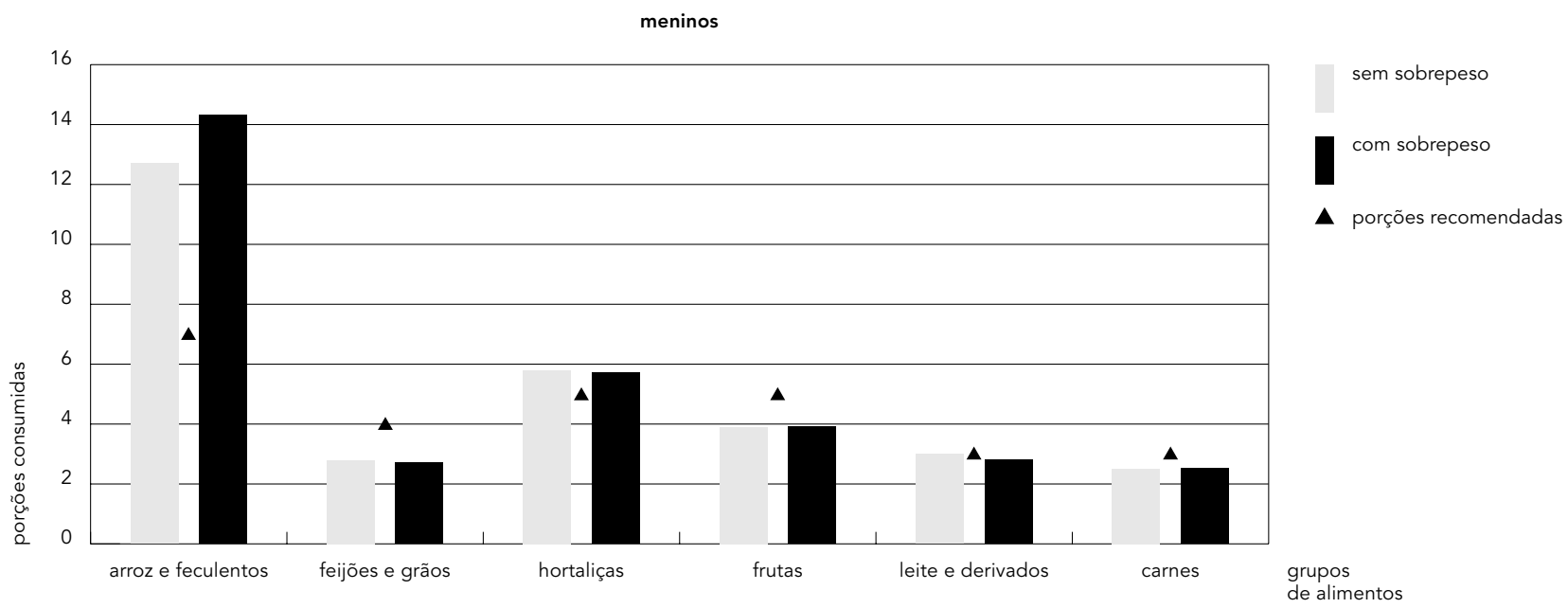

meninas

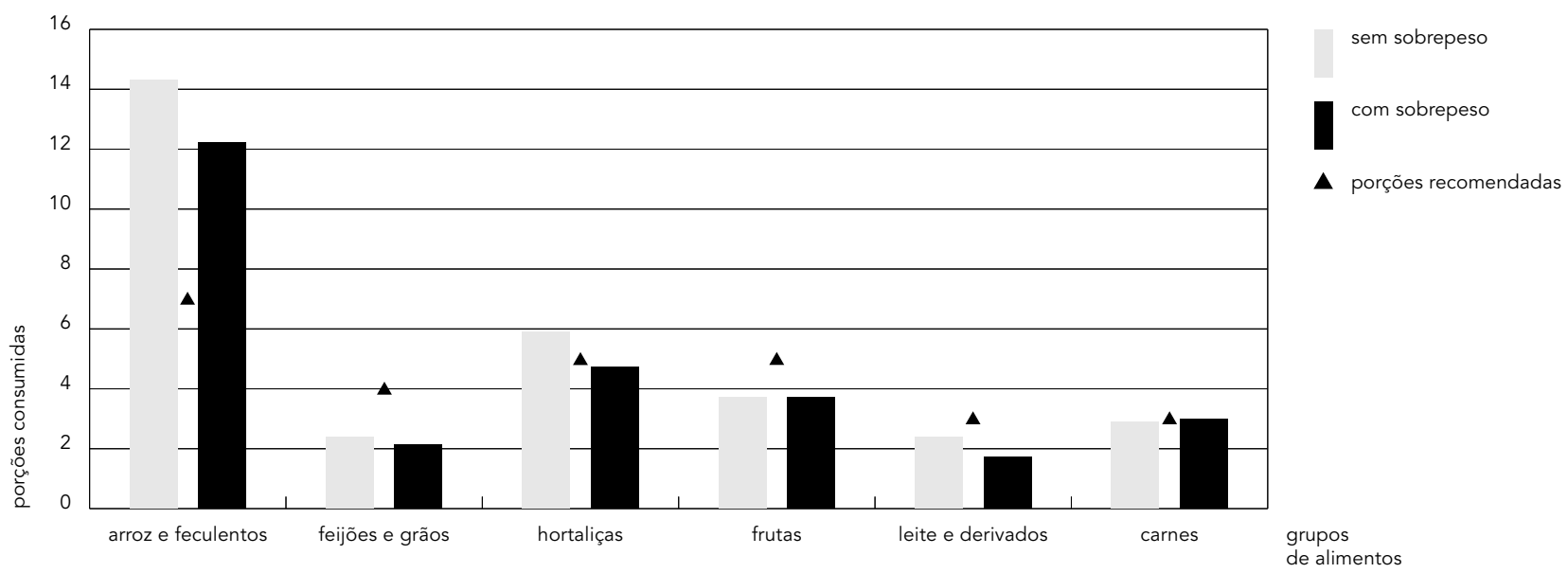

mente entre as meninas com sobrepeso, a participação das batatas fritas foi aproximadamente o dobro da observada entre os meninos.

Na população brasileira, em curto espaço de tempo, observou-se um incremento de prevalência de obesidade, que embora tenha sido acompanhado de mudanças no perfil de alimentação, não parece ter se acompanhado de uma elevação significativa de consumo de energia (Sichieri et al., 1997). Segundo teorias recentes, a restrição alimentar na infância poderia facilitar o excesso de peso na vida adulta. Assim sendo, pequenas variações de consumo que se- riam de difícil mensuração poderiam gerar um superávit de energia a ser estocado.

No Brasil, até três décadas atrás, a prevalência da desnutrição era elevada (Monteiro et al., 2000a). Estudos recentes mostraram que em adolescentes que sofreram desnutrição pregressa a taxa de oxidação de gordura era superior à observada em adolescentes eutróficos. Essa seria uma característica metabólica que predisporia o organismo ao excesso de peso (Hoffman et al., 2000). Por outro lado, em um estudo longitudinal de três anos, observou-se que meninas entre 8 e 11 anos que sofreram 
desnutrição pregressa tinham taxa de metabolismo de repouso menor e velocidade de ganho de peso maior do que as meninas eutróficas (Hoffman et al., 2000).

Portanto, a desnutrição na infância associada ao excesso de peso é uma hipótese que não pode ser excluída para um grupo significativo dos indivíduos estudados, o que, também, poderia estar contribuindo para a não distinção entre a dieta de adolescentes com e sem sobrepeso.

Costa \& Sichieri (1998) estudaram o mesmo grupo investigado nesta pesquisa, com o objetivo de avaliar se a obesidade, o sobrepeso e a distribuição de gorduras seriam fatores explicativos para a associação entre o aumento da pressão arterial e o crescimento, nele as variáveis indicadoras de sobrepeso associaram-se com a pressão arterial, particularmente entre as meninas, sugerindo que o controle de peso em idades precoces possa ter impacto sobre a prevalência de hipertensão arterial essencial do adulto.

A importância da dieta em períodos precoces da vida tem sido salientada em inúmeras investigações (Barros \& Victora, 1999; Ebrahim, 1997; Maheswaran et al., 1997; Smith \& Shlomo, 1997). A dieta inadequada na adolescência pode ser um fator de risco para enfermidades,

\section{Referências}

BARROS, F. C. \& VICTORA, C. G., 1999. Increased blood pressure in adolescents who were small for gestational age at birth: A cohort study in Brazil. International Journal of Epidemiology, 28:676681.

COITINHO, D. C.; LEÃO, M. M.; RECINE, E. \& SICHIERI, R., 1991. Condições Nutricionais da População Brasileira. Pesquisa Nacional sobre Saúde e Nutrição. Brasília: Instituto Nacional de Alimentação e Nutrição, Ministério da Saúde.

COSTA, R. S. \& SICHIERI, R., 1998. Relação entre sobrepeso, adiposidade e distribuição de gordura com a pressão arterial de adolescentes no Município do Rio de Janeiro. Revista Brasileira de Epidemiologia, 1:268-279.

EBRAHIM, S., 1997. Stroke mortality - Secular and geographic trends: Comment on papers by Maheswaran and colleagues. Journal of Epidemiology and Community Health, 51:132-133.

ENGSTROM, E. M.; SILVA, D. E.; ZABOROWSKI, E. L.; BARROS, D. C. \& MONTEIRO, K. A., 1998. Diagnóstico Nutricional de Adolescentes e escolares. In: Sisvan: Instrumento para o Combate aos distúrbios Nutricionais em Serviços de Saúde - O Diag- particularmente as de origem metabólica, na vida adulta. Os eventos nutricionais na vida precoce podem determinar alterações nos sistemas anabólico/catabólico, levando a desfechos metabólicos em idades mais avançadas e permitindo a acumulação de tecido adiposo mediante modificações muito pequenas no consumo energético (Sichieri et al., 1997). Dessa forma, pode ser elevada a probabilidade de adolescentes com hábitos alimentares inadequados tornarem-se adultos obesos e candidatos às doenças crônicas não transmissíveis.

Fase de construção da identidade do sujeito, a adolescência é um momento privilegiado para as intervenções na área da saúde e da nutrição tendo em vista a adoção de hábitos de vida saudáveis e a promoção da saúde na vida adulta. Nesta fase, o indivíduo adquire comportamentos que, em grande parte, vai manter ao longo de toda a vida (Engstrom et al., 1998).

Embora a prevalência de sobrepeso entre adolescentes no Brasil ainda esteja distante dos índices encontrados em outros países como, por exemplo, os Estados Unidos, a tendência de elevação destas taxas (SMS/SME, 2000) reitera a necessidade de desenvolver projetos de prevenção, particularmente via uma alimentação saudável. nóstico Nutricional (E. M. Engstrom, D. E. Silva, E. L. Zaborowski, D. C. Barros \& K. A. Monteiro, org.), pp. 79-88, Rio de Janeiro: Editora Fiocruz.

EPM (Escola Paulista de Medicina), 1995. Programa de Apoio à Decisão em Nutrição, Versão 2.5. São Paulo: Escola Paulista de Medicina, Universidade Federal de São Paulo.

IBGE (Fundação Instituto Brasileiro de Geografia e Estatística), 1985. Estudo Nacional de Despesa Familiar - ENDEF Tabela de Composição de Alimentos. Rio de Janeiro: IBGE.

FONSECA, V. M.; VEIGA, G. V. \& SICHIERI, R., 1998. Fatores associados à obesidade em adolescentes. Revista de Saúde Publica, 32:541-549.

FUJIMORI, E.; SZARFARC, S. C. \& OLIVEIRA, I. M. V., 1996. Prevalência de anemia e deficiência de ferro em adolescentes do sexo feminino: Taboäo da Serra, SP, Brasil. Revista Latinoamericana de Enfermagem, 4:49-63.

GONÇALVES-CARVALHO, C. M. R.; AMAYA-FARFAN, B. C. W. \& VENCONVSKY, R., 1995. Prevalência de hipovitaminose A em crianças da periferia do Município de Campinas, São Paulo, Brasil. Cadernos de Saúde Pública, 11:85-96. 
HELSING, E., 1995. Traditional diets and disease patterns of the Mediterranean, circa 1960. American Journal of Clinical Nutrition, 61:1329S-1337S.

HIMES, J. H. \& DIETZ, W. H., 1994. Guidelines for overweight in adolescent preventive services: Recommendations from an expert committee. The Expert Committee on Clinical Guidelines for Overweight in Adolescent Preventive Services. American Journal of Clinical Nutrition, 59:307-316.

HOFFMAN, D. J.; SAWAYA, A. L.; VERRESCHI, I.; TUCKER, K. L. \& ROBERTS, S. B., 2000. Why are nutritionally stunted children at increased risk of obesity? Studies of metabolic rate and fat oxidation in shantytown children from São Paulo, Brazil. American Journal of Clinical Nutrition, 72:702-707.

JACOBSON, M. S., 2001. Nutrição na adolescência. Anais Nestlé, 55:24-33.

LOHMAN, T. G.; ROCHE, A. F. \& MARTORELL, R., 1988. Anthropometrics Standardization Reference Manual. Champaign: Human Kinetics Books.

MAHESWARAN, R.; ELLIOT, P. \& STRACHAN, D. P., 1997. Socioeconomic deprivation, ethnicity, and stroke mortality in greater London and southeast England. Journal of Epidemiology and Community Health, 51:127-131.

MONDINI, L. \& MONTEIRO, C. A., 1994. Mudanças no padrão de alimentação da população urbana brasileira (1962-1988). Revista de Saúde Pública, 28:433-439.

MONTEIRO, C. A.; BENÍCIO, M. H. A.; IUNES, R. F.; GOUVEIA, N. C. \& CARDOSO, M. A. A., 2000a. Evolução da desnutrição infantil. In: Velhos $e$ Novos Males da Saúde no Brasil - A Evolução do País e de suas Doenças (C. A. Monteiro, org.), pp. 93-114, São Paulo: Editora Hucitec/Núcleo de Pesquisas Epidemiológicas em Nutrição e Saúde, Universidade de São Paulo.

MONTEIRO, C. A.; MONDINI, L. \& COSTA, R. B. L., $2000 \mathrm{~b}$. Secular changes in dietary patterns in the metropolitan areas of Brazil (1988-1996). Revista de Saúde Pública, 34:251-258.

ORTEGA, M. R.; REQUEJO, A. M.; ANDRES, P.; LOPEZSOBALER, A. M.; REDONDO M. R. \& GONZALEZFERNANDEZ, M., 1995. Relationship between diet composition and body mass index in a group of Spanish adolescents. British Journal of Nutrition, 74:765-773.

PENNINGTON, J. A. T., 1989. Food Values of Portions Commonly Used. 15 a Ed. New York: Perennial Library/Harper \& Row.

PEREIRA, R. A., 1998. Avaliação antropométrica do estado nutricional. In: Epidemiologia da Obesidade (R. Sichieri, org.), pp. 43-64, Rio de Janeiro: Eduerj.

ROLLS, B. J.; BEEL, E. A.; CASTELLANOS, V. H.; CHOW, M.; PELKMAN, C. L. \& THORWART, M. L., 1999. Energy density not fat content of foods affected intake in lean and obese women. American Journal of Clinical Nutrition, 69:863-871.

SMS (Secretaria Municipal de Saúde)/SME (Secretaria Municipal de Educação), 2000. II Pesquisa de Saúde e Nutrição em Escolares, RJ. Rio de Janeiro: SMS/SME.

SICHIERI, R., 1998. Avaliação do consumo alimentar e do consumo de energia. In: Epidemiologia da
Obesidade (R. Sichieri, org.), pp. 65-88, Rio de Janeiro: Eduerj.

SICHIERI, R. \& ALLAM, V. C., 1996. Avaliação do estado nutricional de adolescentes brasileiros através do índice de massa corporal. Jornal de Pediatria, 72:80-84.

SICHIERI, R.; COITINHO, D.; PEREIRA, R. A.; COUTINHO, D. C.; MARINS, V. M. R. \& MOURA, A. S., 1997. Variações temporais do estado nutricional e do consumo alimentar no Brasil. Physis, 7:3135.

SICHIERI, R. \& EVERHART, J. E., 1998. Validity of a Brazilian food frequency questionnaire against dietary recalls and estimated energy intake. $\mathrm{Nu}$ trition Research, 18:1649-1659.

SICHIERI, R. \& SILVA, N. N., 1998. Metodologia da pesquisa nutrição e saúde no Município do Rio de Janeiro. In: Epidemiologia da Obesidade (R. Sichieri, org.), pp. 35-42, Rio de Janeiro: Eduerj.

SICHIERI, R.; COITINHO, D. C.; BRESSAN, J. B. \& COUTINHO, W. F., 2000. Recomendações de alimentação e nutrição saudável para a população brasileira. Arquivos Brasileiros de Endocrinologia e Metabologia, 44:227-232.

SMITH, G. D. \& BEN-SHLOMO, Y., 1997. Geographical and social class differentials in stroke mortality - The influence of early-life factors: Comment on papers by Maheswaran and colleagues. Journal of Epidemiology and Community Health, 51: 134-137.

STORY, M.; TOMPKINS, R. A.; BASS, M. A. \& WAKEFIELD, L. M., 1986. Anthropometric measurements and dietary intakes of Cherokee Indian teenagers in North Carolina. Journal of American Dietetic Association, 86:1555-1560.

SUMMERBELL, C. D.; MOODY, R. C.; STOCK, M. J.; SHANKS, J. \& GEISSLER, C., 1996. Relationship between feeding pattern and body mass index in 220 free-living people in four age groups. European Journal of Clinical Nutrition, 50:513-519.

VEIGA, G. V., 1992. Contribuição ao Estudo da Obesidade em Adolescentes do sexo Feminino: Uma Avaliação Comparativa entre dois Níveis Sócioeconômicos. Tese de Doutorado, São Paulo: Escola Paulista de Medicina, Universidade Federal de São Paulo.

VEIGA, G. V.; DIAS, P. C. \& ANJOS, L. A., 2001. A comparison of distribution curves of body mass index from Brazil and the United States for assenssing overweight and obesity in Brazilian adolescents. Revista Panamericana de Salud Pública, 10:79-84.

WELSH, S.; DAVIS, C. \& SHAW, A., 1992. Development of the food guide pyramid. Nutrition Today, 27: 12-23.

WHITEHEAD, R. G. \& PAUL, A. A., 1995. Estudo longitudinal sobre as necessidades energéticas de crianças e adolescentes. In: A Alimentação da Idade Pré-Escolar até a Adolescência, Brochuras Nestlé 37, Resumos, pp. 1-3, Madrid: Nestlé.

Recebido em 7 de novembro de 2002

Versão final reapresentada em 9 de abril de 2003 Aprovado em 21 de julho de 2003 